\title{
Profiles of relative permittivity and electrical conductivity from unsaturated soil water content models
}

\author{
Roberta Porretta ${ }^{\star}$, Fabio Bianchi
}

Istituto Nazionale di Geofisica e Vulcanologia, Rome, Italy

\author{
Article history \\ Received February 19, 2016; accepted May 17, 2016. \\ Subject classification: \\ Soil texture, Diffusion, Water content, Conductivity/permittivity profiles.
}

\begin{abstract}
A mathematical model of water diffusion in the vadose zone has been implemented for different types of soil textures in order to determine the soil water content (SWC) profiles in dependence of depth and time. From these profiles, obtained for different soils, we derived the characteristic electrical parameters, such as relative permittivity $\left(\varepsilon_{r}\right)$ and electrical conductivity $(\sigma)$, and their variation in time, employing empirical relations available in the scientific literature. The simulation through mathematical models has been performed taking into account different types of soils characterized by the percentage composition of sand, clay and silt in the textural triangle, which provides some physical and chemical properties that affect the water retention in the soil. The resulting simulated profiles of SWC and consequently permittivity and conductivity profiles, span over a certain range of values suggesting the best techniques and the limits in geophysical investigation. Moreover this a-prior knowledge helps in the elaboration and interpretation of permittivity and conductivity data obtained by the measurements. Permittivity and conductivity profiles are particularly useful in some environmental applications when the soil textures are (or supposed to be) known as in the typical case of landfill leachate dispersion. Since the soil textures beneath or nearby a disposal waste are characterized by a $S W C$, the values of $\varepsilon_{r}$ and $\sigma$ at various depth can be directly inferred.
\end{abstract}

\section{Introduction}

Electrical conductivity $(\sigma)$ and relative dielectric permittivity $\left(\varepsilon_{r}\right)$ are two independent physical properties that characterize the behavior of soil when excited by electric currents or electromagnetic fields [Settimi 2011]. The electrical conductivity and dielectric permittivity of the constituent minerals are the main controlling factors of the electrical properties of soils [Kriaa et al. 2014]. The derivation of these quantities related to the water content provides useful information regarding the practical use of geophysical investigation (GPR, TDR, etc.), applied to unsaturated subsurface vadose zone of medium. This zone has relevant importance in different ambits and especially in geophysical prospection [Huisman et al.
2003]. The unsaturated portion of soil concerns the first meters beneath the ground level down to the water table, where the saturated zone begins. In this section hydrogeological properties (physical and chemical) are important since they dominate the electric properties of soil, described by the two quantities $\sigma$ and $\varepsilon_{r}$. These last quantities can be directly measured through several techniques or theoretically deduced (constitutive relations) in a homogeneous medium. In this paper these parameters are estimated through soil hydrologic models [Huisman et al. 2003]. In the latter case the SWC in vadose zone plays an important role to determine both relative permittivity and electrical conductivity since they are strongly dependent on it. The different soil textures can retain different percentage of water, due to its porosity, under the action of two agents: the gravity potential and the matrix potential. The diffusive equation is driven by these quantities and cannot be solved without models. These models need in input physical parameters that quantify the textures of soil. In the past decades authors proposed different numerical parameters [Mualem 1976, van Genuchten 1980] to solve the so-called Richards equation that well describes the water diffusion in the vadose zone. Once the input parameters for the particular type of soil are established it is possible to numerically calculate the SWC, which is function of time and depth. SWC is the most important hydro-geological parameters that jointly to salinity and porosity affects the electrical properties in the unsaturated soils.

This paper deals with simulations of soil model in order to evaluate the SWC and consequently through Topp and Archie empirical relationships to determine the electrical parameters of soil. In this specific case, we obtained 3D profiles that show temporal and spatial variation of the electrical properties. The study focuses on soil investigation theoretical approach, that could be a useful tool in some environmental and geophysi- 


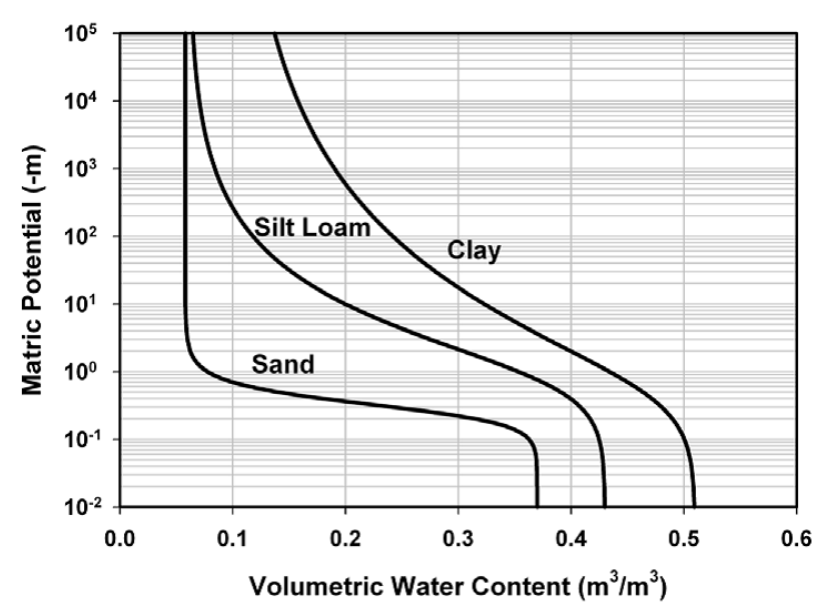

Figure 1. Water retention curve that links the two variables $\theta$ or $\psi$ depending on the type of soil [Tuller and Or 2005].

cal applications. Furthermore it could represent a preliminary phase which precedes the measurements and facilitates the interpretation of the results (GPR, TDR, ERT and other similar geophysical investigation techniques e.g. after raining survey or in landfill control).

\section{Water content models}

Water flows in saturated porous medium and water content retention in different kind of soils has been subject of many studies. To describe this phenomenon in the vadose zone Richards proposed an equation which is a combination between the flux equation and mass conservation, and it characterizes the SWC trend over time [Richards 1931]. The software developed to derivate SWC presented in this paper it's based on well-known literature models such as van Genuchten, Mualem, Brooks and Corey. The introduction of these models was necessary, due to the impossibility to analytically solve the Richards' equation.

The water diffusion equation into unsaturated soil in one-dimensional terms can assume the following form:

$$
\frac{\partial \theta}{\partial t}=\frac{\partial}{\partial z}\left(K \frac{\partial H}{\partial z}\right)
$$

where $\theta$ is the SWC $\left[\mathrm{m}^{3} / \mathrm{m}^{3}\right], H$ represents the hydraulic head: $H=\psi+z$, being $\psi$ the matrix potential and $z$ the elevation above a reference plan (level). $K$ is hydraulic conductivity in $(\mathrm{m} / \mathrm{s})$. The above is the socalled Richard's equation that has no closed form solution because $K$ is depend dependent on $\theta$.

The substitution of the hydraulic head $H$ with $\psi+$ $z$, leads to the so-called Richard's mixed form:

$$
\frac{\partial \theta}{\partial t}=\frac{\partial}{\partial z}\left[K\left(\frac{\partial \Psi}{\partial z}+\frac{\partial z}{\partial z}\right)\right]=\frac{\partial}{\partial z}\left[K\left(\frac{\partial \Psi}{\partial z}+1\right)\right]
$$

Here please note that $K$ is the hydraulic conductivity in $[\mathrm{cm} /$ day $]$ and $\theta$ is the soil water content $\left[\mathrm{cm}^{3} / \mathrm{cm}^{3}\right]$. It describes the movement of the fluid (fluid motion) in a non-saturated and porous medium. It is a non linear partial differential equation and, as such, it has no analytical closed form solution and must be integrated numerically.

$$
\frac{\partial \theta}{\partial t}=\frac{\partial}{\partial z}\left[K(\theta)\left(\frac{\partial \Psi}{\partial z}+1\right)\right]
$$

Furthermore, $K(\theta)$ is not a constant in the strict sense, as it depends on the same $\theta$, hence the problem need an appropriate model to be treated. Here it is presented in one-dimensional term along the vertical $z$. Equations (2) and (3) have a two dependent variables $\theta$ and $H$ (or $\psi$ ), so the resolution of the two forms requires the definition of a constitutive equation that binds $\theta$ and $\psi$ as in Figure 1 [Mualem 1976]. From USDA (United States Departement of Agriculture) triangle textures shown in Figure 2 typical van Genuchten parameters are reported in Table 1 [Leij et al. 1996].

As mentioned earlier $K$ is not a constant, because of its dependence on $\theta$ and for this reasons, a model for each different soil textures is required. This demands a constitutive equation that takes into account the water retention curve.

Such a curve depends on the soil pososity, or, even better depends on the effective saturation $\mathrm{Se}$ [Leij et al. 1997]. The latter can be expressed as:

$$
S e=(\theta-\theta r) /(\theta s-\theta r)
$$

where: $\theta s$ is the water content in saturated conditions and $\theta r$ is the residual water content. It means that if $S e=0$ the fluid can be found only in gaseous phase,

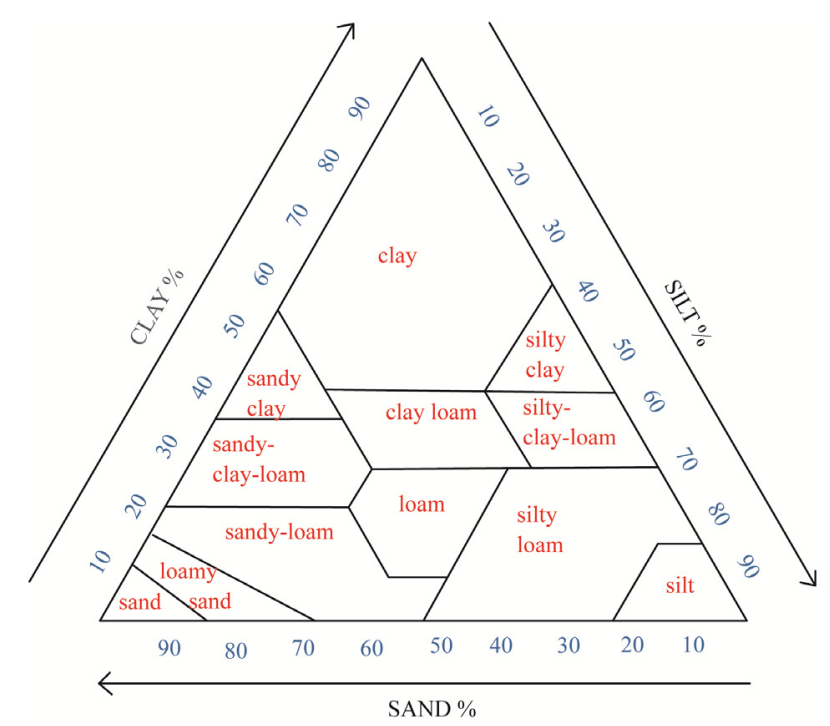

Figure 2. USDA (United States Departement of Agriculture) textures triangle. 


\begin{tabular}{lccccc}
\hline Textural class & $\mathbf{N}$ & $\begin{array}{c}\theta \mathbf{r} \\
{\left[\mathbf{c m}^{3} / \mathbf{c m}^{3}\right]}\end{array}$ & $\begin{array}{c}\theta \mathbf{s} \\
{\left[\mathbf{c m}^{3} / \mathbf{c m}^{3}\right]}\end{array}$ & $\begin{array}{c}\alpha \\
{[\mathbf{1} / \mathbf{c m}]}\end{array}$ & $\mathbf{n}$ \\
\hline Sand & 126 & 0.058 & 0.37 & 0.035 & 3.19 \\
Loamy sand & 51 & 0.074 & 0.39 & 0.035 & 2.39 \\
Sandy loam & 78 & 0.067 & 0.37 & 0.021 & 1.61 \\
Loam & 61 & 0.083 & 0.46 & 0.025 & 1.31 \\
Silt & 3 & 0.123 & 0.48 & 0.006 & 1.53 \\
Silt loam & 101 & 0.061 & 0.43 & 0.012 & 1.49 \\
Sandy clay loam & 37 & 0.086 & 0.40 & 0.033 & 1.37 \\
Clay loam & 23 & 0.129 & 0.47 & 0.030 & 1.41 \\
Silty clay loam & 20 & 0.098 & 0.55 & 0.027 & 1.39 \\
Silty clay & 12 & 0.163 & 0.47 & 0.023 & 1.20 \\
Clay & 25 & 0.102 & 0.51 & 0.021 & \\
\hline
\end{tabular}

Table 1. Typical van Genuchten model with parameters $(\alpha, n)$ including residual $(\theta r)$ and saturated $(\theta s)$.

while if $S e=1$ we are in saturated conditions. Because the hydraulic conductivity $K$ depends on Se, therefore the introduction of models [Brooks and Corey 1964, Mualem 1976] that take into account such a dependence $K \rightarrow K(S e)$ is necessary. In this work the following model proposed by van Genuchten has been adopted:

$$
K\left(S_{e}\right)=K \sqrt{S_{e}\left[1-\left(S_{e}^{1 / m}\right)^{m}\right]^{2}}
$$

Such a model [van Genuchten 1980] is widely employed in this field. In this work was also introduced the dependence on the matrix potential like:

$$
S e=1 /\left(1+|\alpha \psi|^{n}\right)^{m}
$$

with tabulated parameters $\alpha, n=1-1 / m$

$$
\frac{\partial \theta}{\partial \Psi}=\frac{\left(\theta_{s}-\theta_{r}\right) n m \alpha(-\alpha h)^{n-1}}{\left(1+|\alpha \psi|^{n}\right)^{m+1}}
$$

Equation (3) after some few arrangements becomes

$$
\frac{\partial \theta}{\partial \Psi} \frac{\partial \Psi}{\partial t}=\frac{\partial}{\partial z}\left[K(\theta)\left(\frac{\partial \Psi}{\partial z}+1\right)\right]
$$

Equation (8) is numerically solved in Matlab program through the van Genuchten's model, given in relations (5), (6) and (7). This allows to obtain both $\theta(z, t)$ and $\psi(z, t)$ variables in function of time and depth. Since the implemented model it's based on equations having validity in vadose zone the simulated depth can only reach the water table. Limits on the depth parameter are intrinsic in the matrix potential that cannot act on more than $10 \mathrm{~m}$ above the water table in ideal conditions, which reflects in a $2-5 \mathrm{~m}$ action in real condi- tions depending on soil texture. Also the heterogeneity of the soil has been strongly simplified and the missing data interpolated with a statistical algorithm.

\section{Empirical relations to derive relative permittivity of the soil}

Many physical and empirical models have been suggested for the evaluation of the $\theta-\varepsilon_{r}$ relationship in the literature. The relative permittivity is the main electric quantity employed to define SWC in the soil since it can be easily measured through various techniques. The relation that ties these two quantities was empirically established [Topp et al. 1980]. This equation is valid for a wide range of mineral soils and independent from soil bulk density, ambient temperature, and salt content. This led many authors to the use of the term "universal" for this equation with appropriate caveat that "in organic soils or heavy clay soils problems arise which may require site-specific calibration" [Cosenza et al. 2003, and the reference therein]. In this specific study the Topp's formula [Topp et al. 1980] has been used. From well-known water content profiles, obtained by a modeling software [Bianchi et al. 2015], Topp's relation (9) allows to derivate the relative permittivity values of soil from the SWC. This empirical model was generated using time-domain reflectometry (TDR) at a frequency between $1 \mathrm{MHz}$ and $1 \mathrm{GHz}$ to measure $\varepsilon_{r}$ for several mineral soils. The estimated error in this model is 0.013. General Topp's model can be expressed:

$$
\varepsilon_{r}=3.03+9.3 \theta+146 \theta^{2}-76.7 \theta^{3}
$$

where $\varepsilon_{r}$ is the relative permittivity and $\theta$ is the soil volumetric water content. They also provided another inverse relation as follows: 


$$
\begin{gathered}
\theta w\left(\varepsilon_{r}\right)=-5.3 \times 10^{-2}+2.92 \times 10^{-2} \varepsilon_{r}- \\
5.5 \times 10^{-4} \varepsilon_{r}+4.3 \times 10^{-6} \varepsilon_{r}
\end{gathered}
$$

A specific calibration is needed for soils with higher water content or organic matter:

$$
\varepsilon_{r}=1.74-0.34 \theta+135 \theta^{2}-55 \theta^{3}
$$

for organic soil;

$$
\varepsilon_{r}=3.57-31.7 \theta+11 \theta^{2}-68.2 \theta^{3}
$$

for $450 \mu \mathrm{m}$ glass beds [Mukhlisin and Saputra 2013].

The model relationships works better for frequencies around $100 \mathrm{MHz}$. At higher frequencies and moisture contents near to saturation $(\theta \sim 0.4)$ the Topp-model over-predicts relative permittivity by up to $20 \%$. At very low water contents the Topp-model doesn't perform well, particularly for soils with a large clay content [van Dam et al. 2005]. However, later studies have shown the dependency of the $\theta-\varepsilon_{r}$ relationship on clay content [Persson et al. 2000, Bouksila et al. 2008] and mineralogy [Cosenza and Tabbagh 2004], organic matter and porosity or soil density [Malicki et al. 1996 and Persson et al. 2002], and soluble salt content [Dalton 1992, Nadler et al. 1999, Persson et al. 2000].

Ledieu et al. [1986] have shown another form of empirical equations to describe the $\theta-\varepsilon_{r}$ relationship, that can be used to expand the Topp model for higher water content [van Dam et al. 2005] of the form:

$$
\sqrt{\varepsilon_{r}}=\mathrm{b}_{0}+\mathrm{b}_{1} \theta
$$

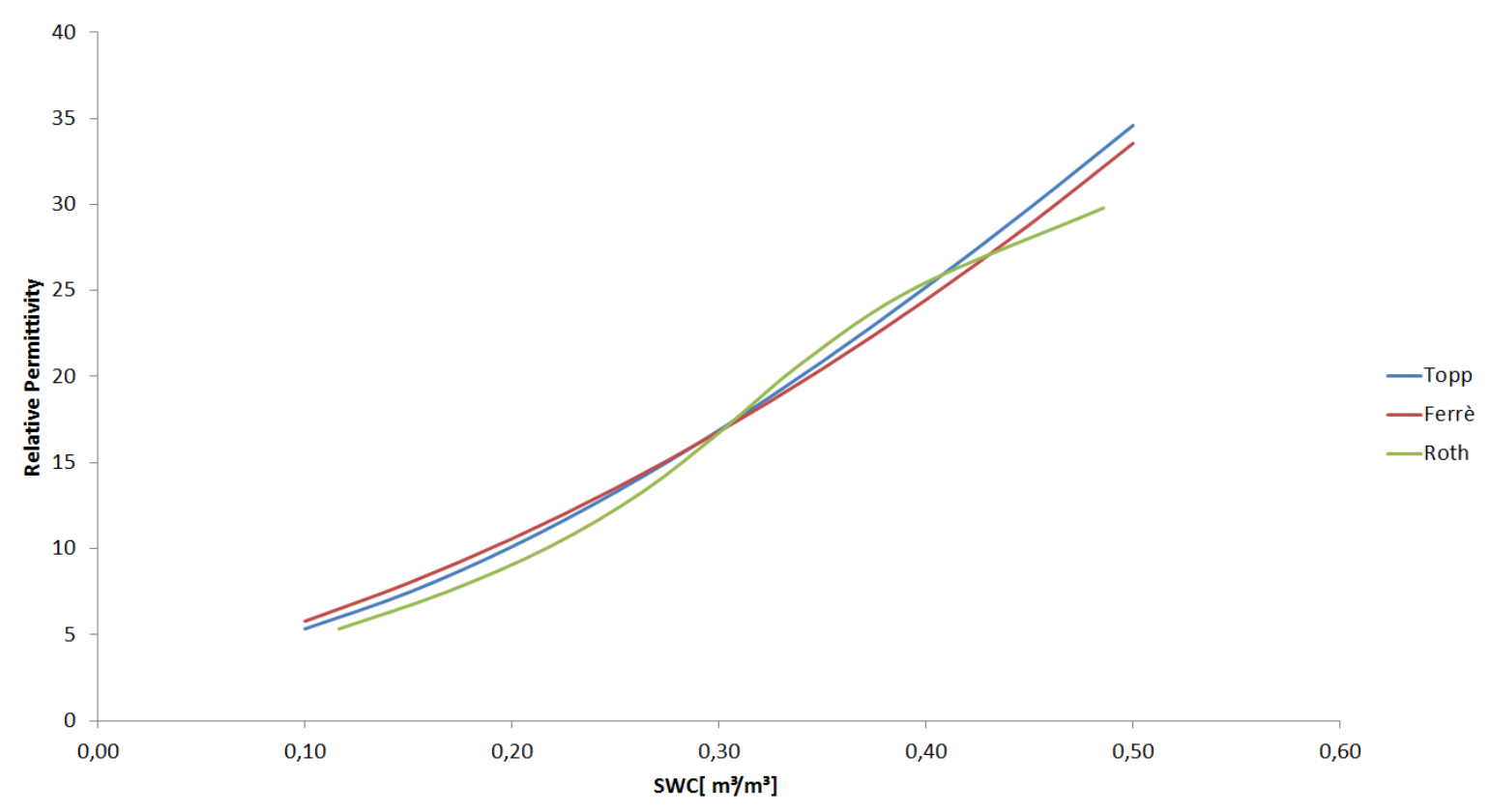

Figure 3. Empirical relations comparison. where $b_{0}$ and $b_{1}$ are two empirical parameters depending on soil type. In the same paper Ledieu et al. [1986] found the following relationship:

$$
\theta=0.1138 \varepsilon_{r}^{0.5}-0.1758
$$

This relationship appears to work better for most mineral non-magnetic soils over a range of frequencies between $1 \mathrm{MHz}$ and $10 \mathrm{GHz}$ [Hamed et al. 2006].

Roth et al. [1990] for mineral soil proposed this empirical relationship:

$\theta=-0.0728+0.044 \varepsilon_{r}-0.00195 \varepsilon_{r}^{2}+0.0000361 \varepsilon_{r}^{3}$

While for organic soil and material is:

$\theta=-0.0233+0.0285 \varepsilon_{r}-0.000431 \varepsilon_{r}^{2}+0.00000304 \varepsilon_{r}^{3}$

the error estimations of these equations for mineral soil and organic soil are 0.015 and $0.035 \mathrm{~cm}^{3} / \mathrm{cm}^{3}$, respectively [Mukhlisin and Saputra 2013].

Although a comparison of all the above relationships showed a similar trend (Figure 3), Topp's formula (blue curve) resulted the relationship that best fitted the input data, since is calibrated for soils that can hold a water content up to $50 \%$. Indeed in unsaturated zone the pores in the soil can be filled not only by water but also by air.

\section{Empirical relations to derive conductivity of the soil}

Another major controlling factor, of the soil electrical proprieties estimated in this study, is electrical 
conductivity. Although usually geoelectric investigations are focused on the measurement of soil resistivity, in this paper conductivity is preferred to resistivity (i.e. the inverse quantity), since prevalently we refer to rain water data given in terms of conductivity entering in the following equations. The empirical relation to estimate the electrical conductivity both for saturated and unsaturated soil is the Archie's law [Shah and Singh 2005] also spread in the literature.

This last one connect electrical conductivity of saturated rocks $\left(\sigma_{o}\right)$ to the conductivity of the electrolyte pore of soils $\left(\sigma_{w}\right)$, it can be expressed as:

$$
\sigma_{o}=\frac{1}{F} \sigma_{w}+\sigma_{q o}
$$

where $\sigma_{q 0}$ is the conductivity contribution which characterizes high clay percentage soils. It can only be neglected for high saline water content filling high porosity soils. $F$ is the formation factor which is equal to:

$$
F=\phi^{-m}
$$

The parameter $\phi$ is the porosity, and $m$ is the cementation exponent which increases with compaction, cementation and consolidation; it varies between 1.3 and 2.5. Unconsolidated sands have values in the range between 1.3 and 1.5 [Dannowski and Yaramanci 1999, and the reference therein]. Generally, the resistivity value is greatly influenced by basic soil characteristics variation such as fraction of solid, air and water. According to [Griffiths and King 1981, Telford et al. 1990], resistivity value was highly influenced by pore fluid and grain matrix of geomaterials. In compact condition, it was found that at high compaction values corresponds lower resistivity value (or higher electrical conductivity). The volumes of pore in compact condition were reduced and cause the current to easily propagate especially during the existing of water [Abidin et al. 2013].

Another form has been proposed, known as Archie's second law:

$$
\sigma_{o}=\frac{1}{F} \frac{1}{I} \sigma_{w}+\sigma_{q o}
$$

which has been developed as an extension for unsaturated rocks and soils including the $I$ factor

$$
I=S w^{-n}
$$

The saturation index I depends on the degree of saturation $S w$ and the saturation exponent $n$. This latter was observed to be about 2 for consolidated rocks and to range from 1.3 to 2 for unconsolidated sands [Lesmes 2005, and the reference therein]. Archie's second law (19) has been chosen and implemented in the modeling software to obtain profiles of electrical conductibility trend over time which characterizes the vadose zone.

This physical quantity depends on a number of parameters as soil texture, water holding capacity, organic matter, salinity and ions exchange capacity. The conductivities of water filling pores is an important factor in the process of electric current flow through the soil; especially when a quantity of salts are dissolved in it.

\section{Simulations and results}

The software estimation of the effective water content, in a porous soil is determined by Richard's equation (Equation 3). The latter is a partial differential equation that can only be solved with the implementation of the van Genuchten soil models. Hence water content is strongly affected by physical property of soil material (porosity, capillarity, etc.) and fluid properties (viscosity). Indeed at equal initial conditions, retention water content only depends on matrix potential in opposition to the effect of gravity, preventing leaching through the ground till the water table. In the performed software simulation, specific initial condition (infiltration velocity), boundary conditions (water table depth, ground level and saturated zone hydraulic head) and simulation parameters as time and depth have been taken into account.

Infiltration is the volume of water passing into the soil per unit of area per unit of time. Simplifying it has

\begin{tabular}{|c|c|c|c|c|c|c|c|}
\hline Soil type & $\begin{array}{c}\text { Porosity } \\
{\left[\mathrm{cm}^{3} / \mathrm{cm}^{3}\right]}\end{array}$ & $\begin{array}{c}\text { Infiltration } \\
\text { velocity } \\
{[\mathrm{cm} / \text { day }]}\end{array}$ & $\begin{array}{c}\sigma \mathbf{w} \\
{[\mathrm{mS} / \mathrm{m}]}\end{array}$ & $\begin{array}{c}\text { Cementation } \\
\text { exponent }\end{array}$ & $\begin{array}{c}\text { Saturation } \\
\text { exponent }\end{array}$ & $\begin{array}{c}\text { Water table } \\
\text { depth } \\
{[\mathrm{cm}]}\end{array}$ & $\begin{array}{l}\text { Time } \\
{[\text { day }]}\end{array}$ \\
\hline Sand & 0.375 & 4.010 & 5 & 1.4 & 2 & 200 & 4 \\
\hline Silt & 0.489 & 1.688 & 5 & 1.4 & 2 & 200 & 4 \\
\hline Clay & 0.459 & 1.603 & 5 & 2.5 & 2 & 200 & 4 \\
\hline
\end{tabular}
the dimensions of velocity $\left[\mathrm{m}^{3} \mathrm{~m}^{-2} \mathrm{~s}^{-1}\right]$. Depth is intended not only as the depth of the water table, or line of saturation, but also the points in the modeled space

Table 2. Simulation parameters. 
where the solution is calculated. Hydraulic head is an equivalent measure of the pressure expressed in height of water column, and it is the sum of pressure head (fluid internal pressure) and elevation head (pressure due to gravity). Following a summarizing Table 2 of the main simulation coefficients, parameters and physical quantities that were used to determinate the electrical conductivity, permittivity and water content relative to the soil textures object of this paper.

The simulation through mathematical models of the WC profiles has been reported, respectively for the most common texture soils types (Figures 4, 5, 6). The picture obtained by the modeling software, shows range of water content up to $50 \%$, and the relative permittivity and electrical conductivity values linked to it, plotted in a 3D space. A time of four days has been chosen because sand soils after that amount of time lose all the water retained in it, and resulted completely dry. Due to this quite fast change in SWC, the electrical properties are also subject to a rather remarkable variation. So the results of the simulation show how taking a measure on a certain soil texture (after-rain survey) could be misleading if the soil retention is not taken in consideration. From Figure 4 it is possible to see how, in a typical clay soil, water content in the topsoil layer changes very little due to its high degree of retention. In the other hand, sand soil (Figure 5) with mediumsize texture have a low moisture holding capacity, which results in a seep through the ground downwards. Silt take up an intermediate behavior between sand and clay soils (Figure 6). Topp's derivated relative permittivity as shown below for clay, sand and silt (Figures 7, $8,9)$ and Table 3 shown an average of relative permittivity values $\left(\varepsilon_{r}\right)$.

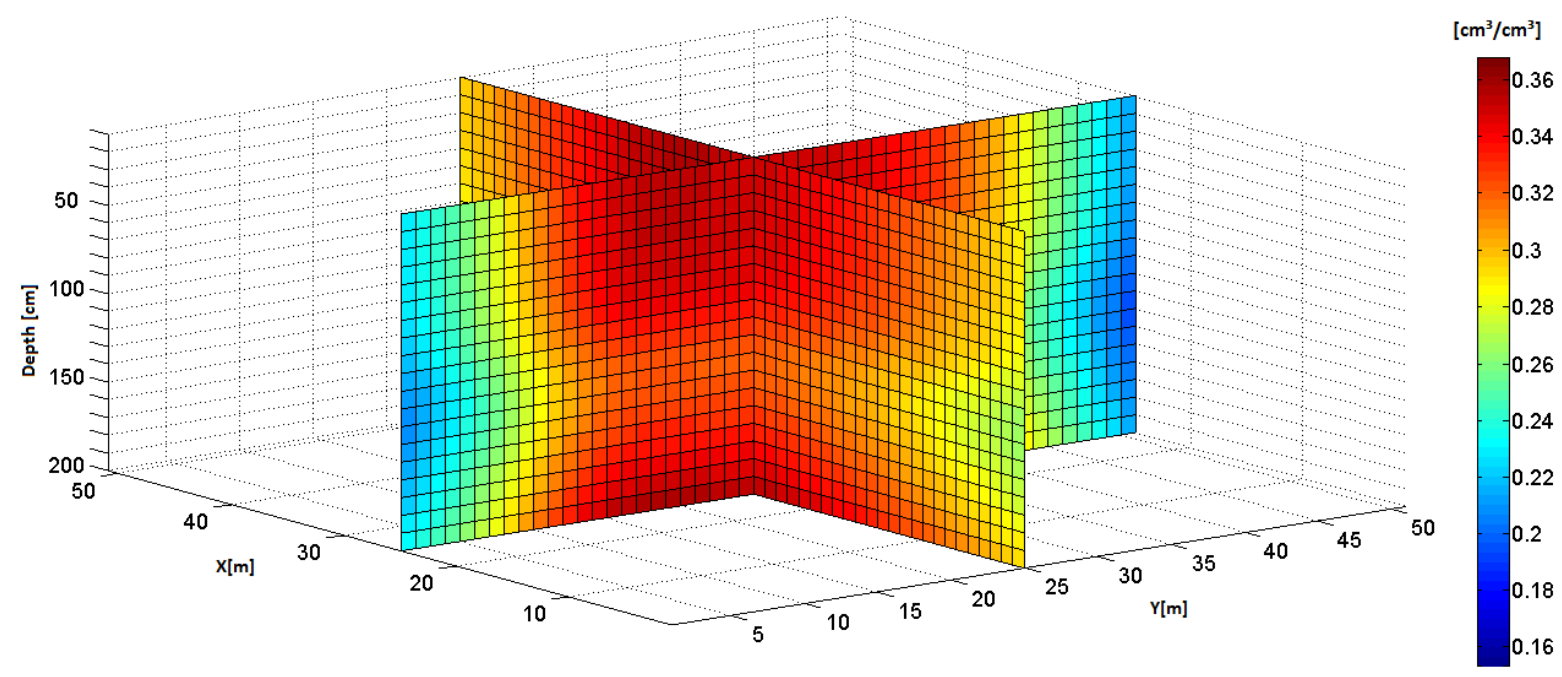

Figure 4. Water content section in clay soil textures.

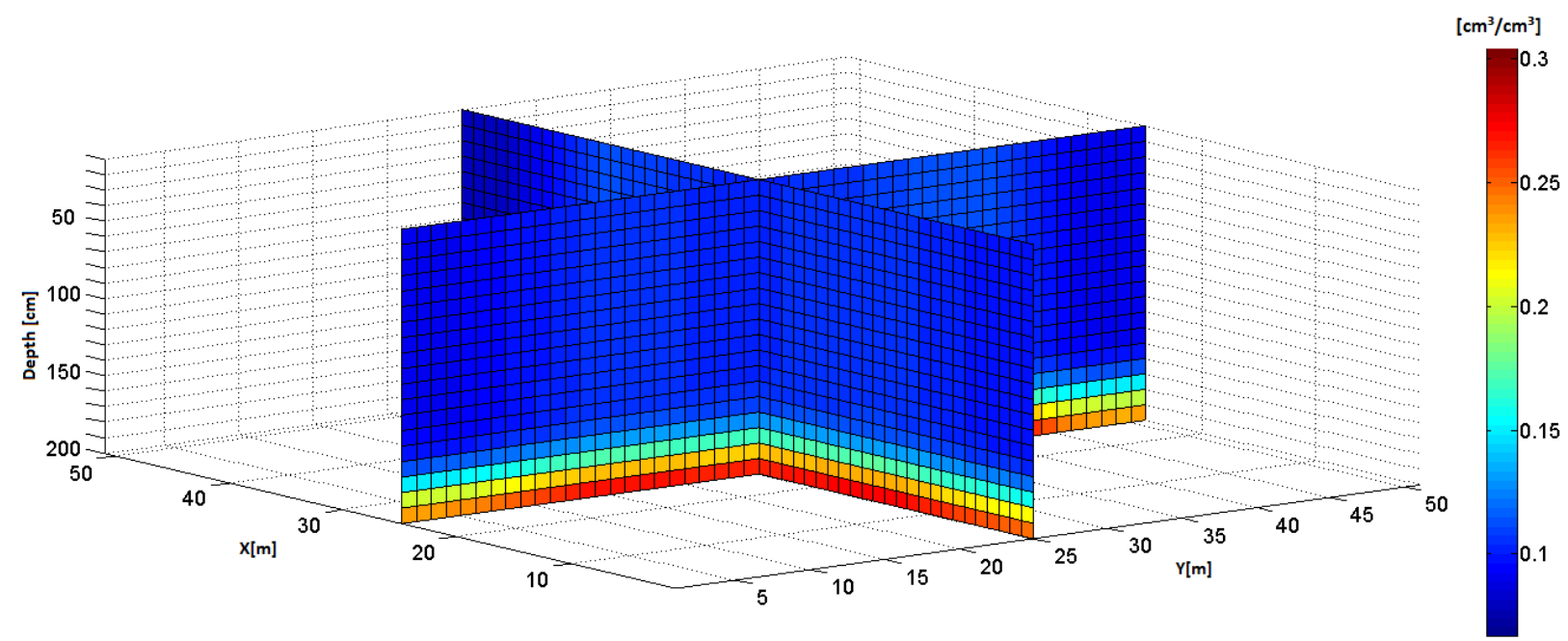

Figure 5. Water content section in sand soil textures. 


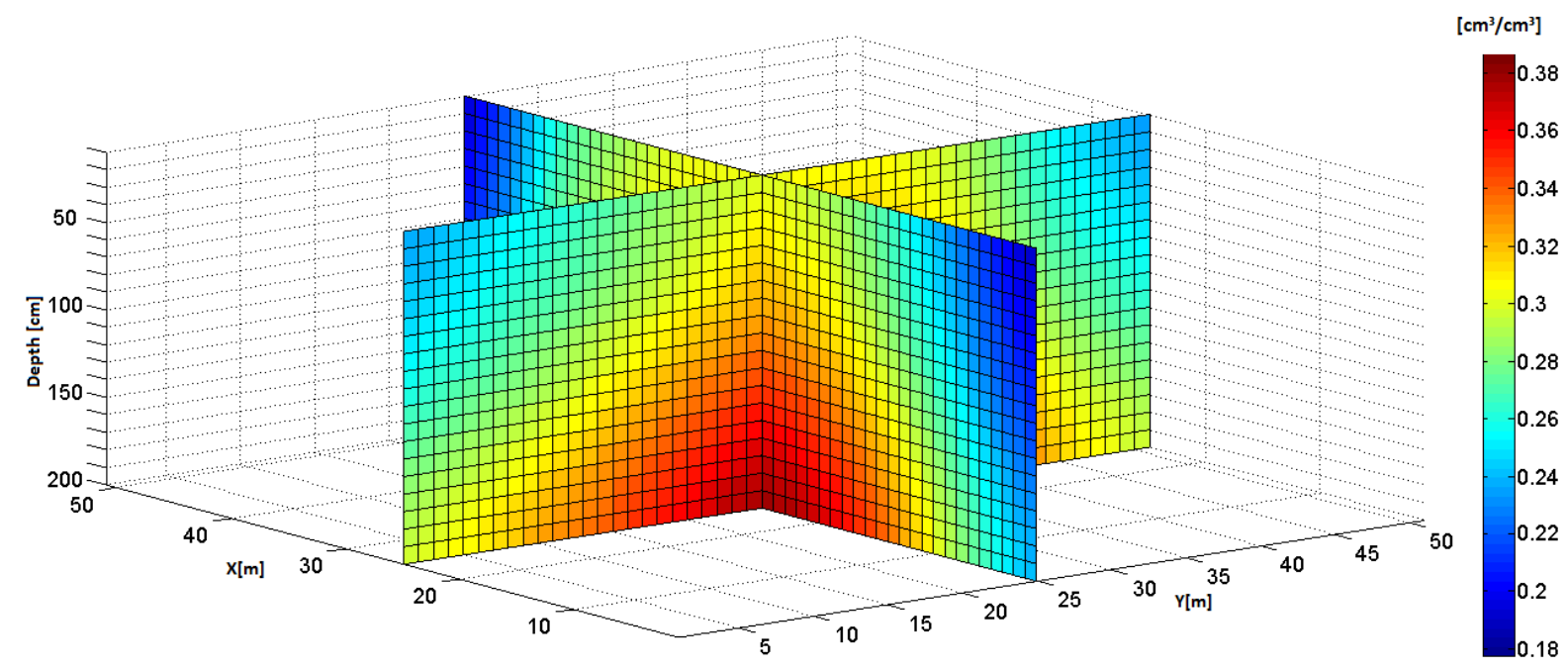

Figure 6. Water content section in silt soil textures.

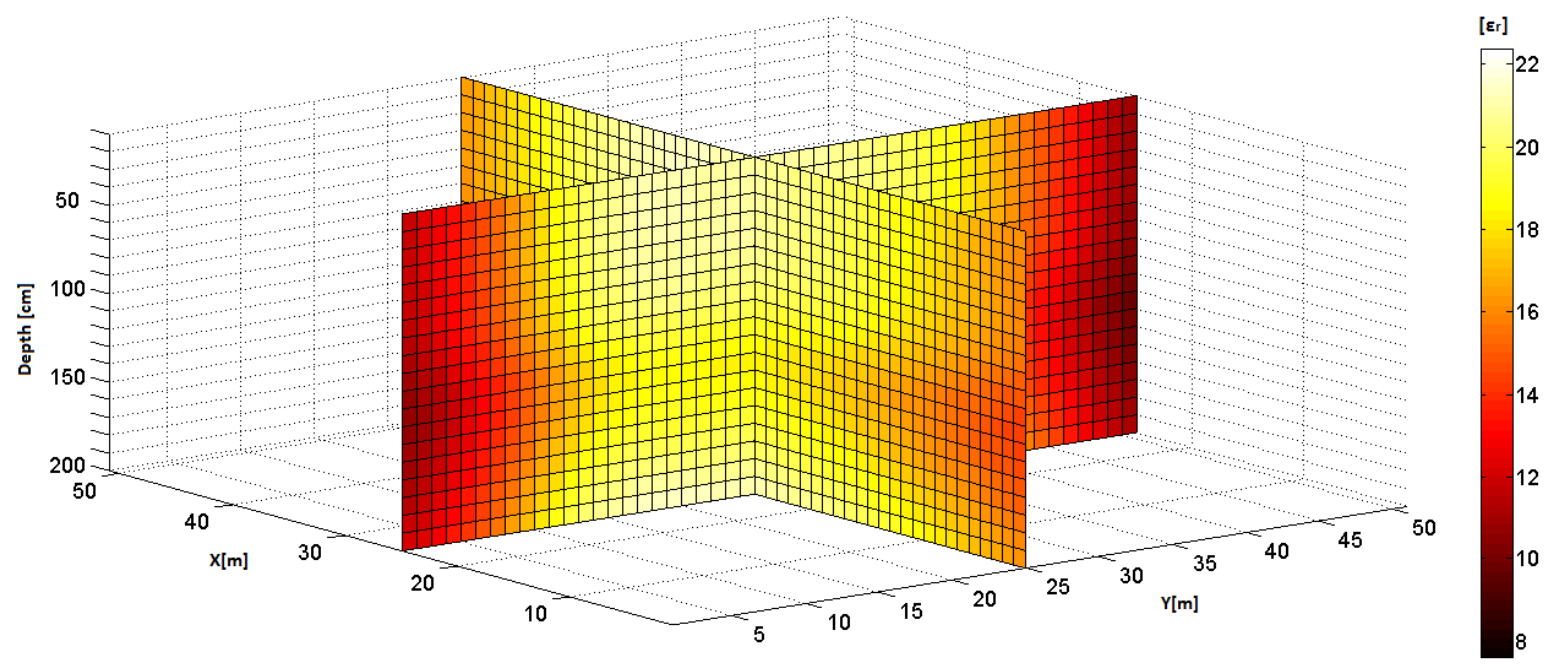

Figure 7. Relative permittivity section in clay soil textures.

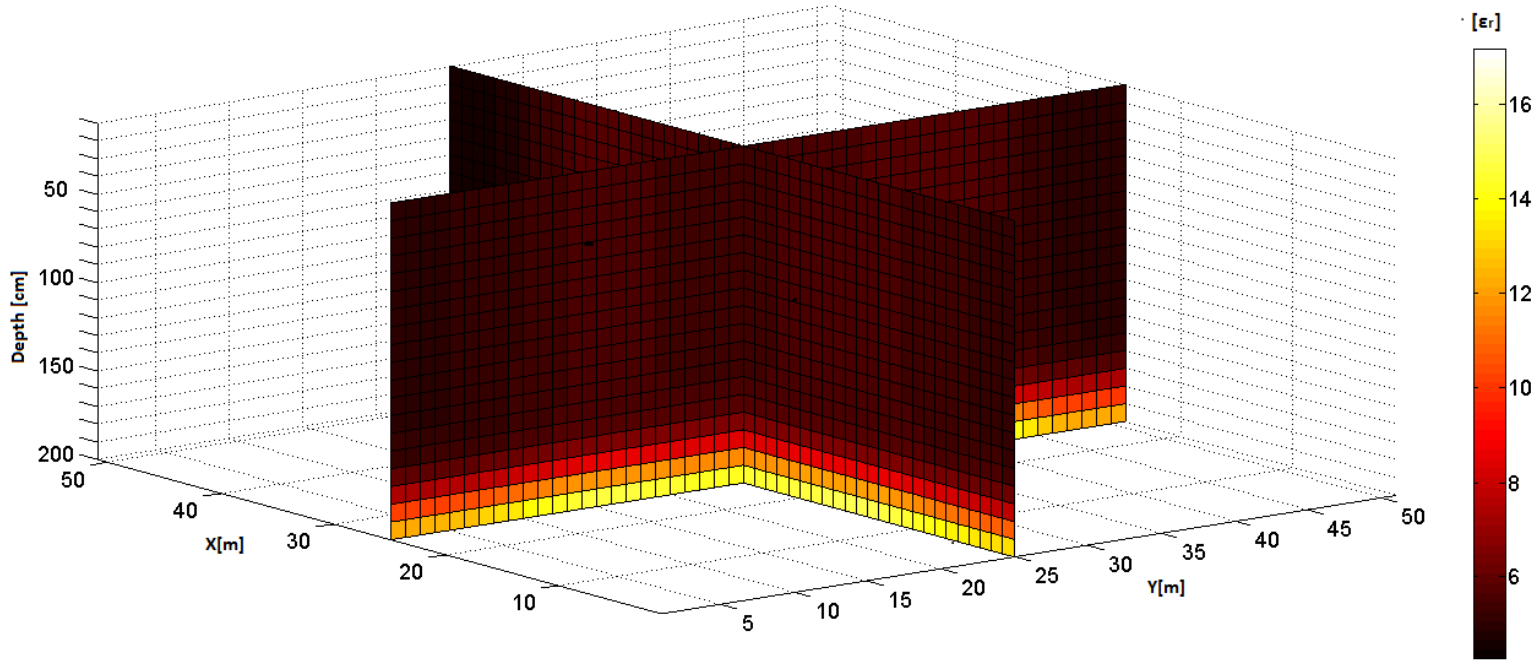

Figure 8. Relative permittivity section in sand soil textures. 


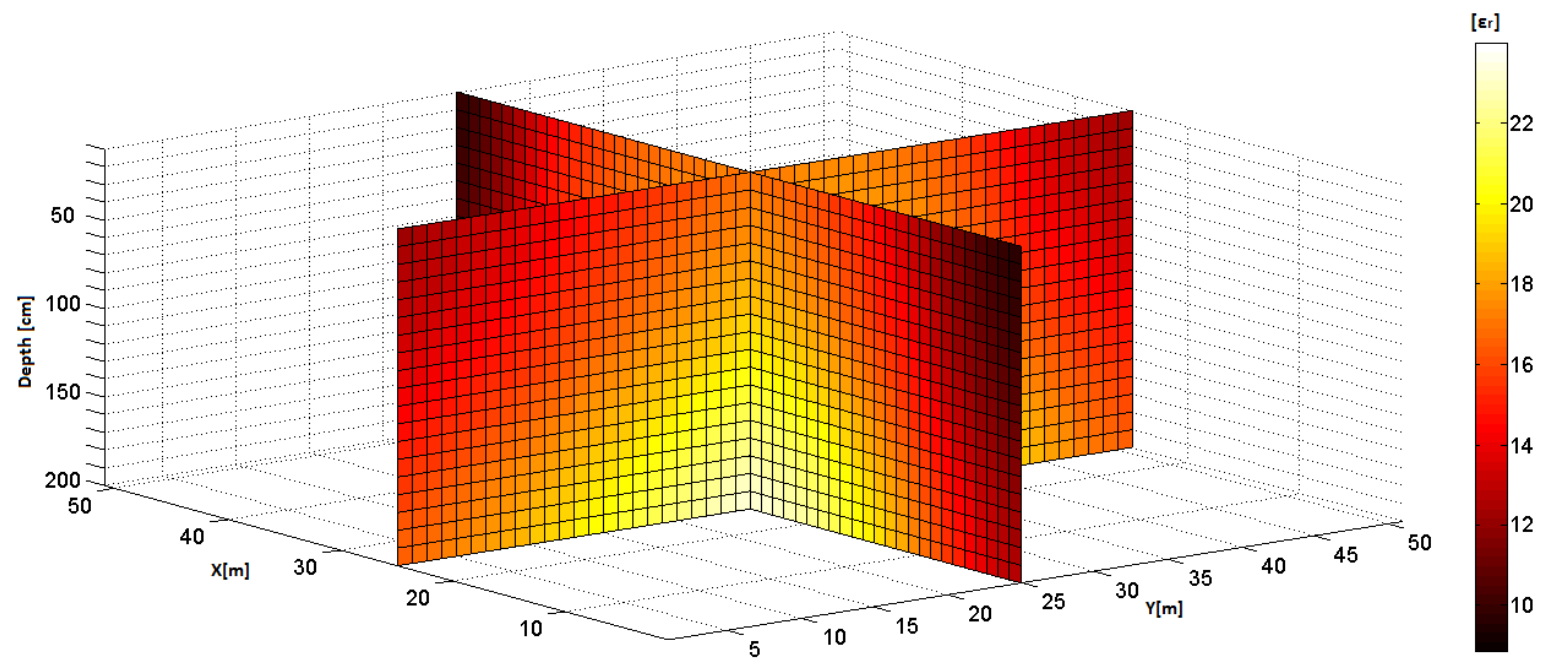

Figure 9. Relative permittivity section in silt soil textures.

\begin{tabular}{|c|c|}
\hline $\begin{array}{c}\boldsymbol{\theta} \\
{\left[\mathrm{cm}^{3} / \mathrm{cm}^{3}\right]}\end{array}$ & $\varepsilon_{r}$ \\
\hline 0.10 & 5.343 \\
\hline 0.15 & 7.451 \\
\hline 0.20 & 10.116 \\
\hline 0.25 & 13.281 \\
\hline 0.30 & 16.889 \\
\hline 0.35 & 20.881 \\
\hline 0.40 & 25.201 \\
\hline 0.45 & 29.791 \\
\hline 0.50 & 34.592 \\
\hline
\end{tabular}

Table 3. Relative Permittivity and corresponding SWC values according to Topp relation.
Since relative permittivity is an electrical physical characteristic which exclusively depends on inner water content, for high degree of moisture content, relative permittivity in simulation test increase.

Indeed, clay soils texture are characterized by higher relative permittivity values than sand soils, which generally shows much lower relative permittivity. Silt soils, show a range of values between 0 and 24 .

Profiles for the electrical conductivity have been present in according to Archie's second law (Equation 19). Also in this case, the profiles were obtained simulating a time of four days as shown in Figures 10, 11 and 12.

Since electrical conductivity is linked to total dissolved solids (TDS) and increase considerably in function of this quantity. To simplify the model, a fixed total solute concentration has been considered, which results

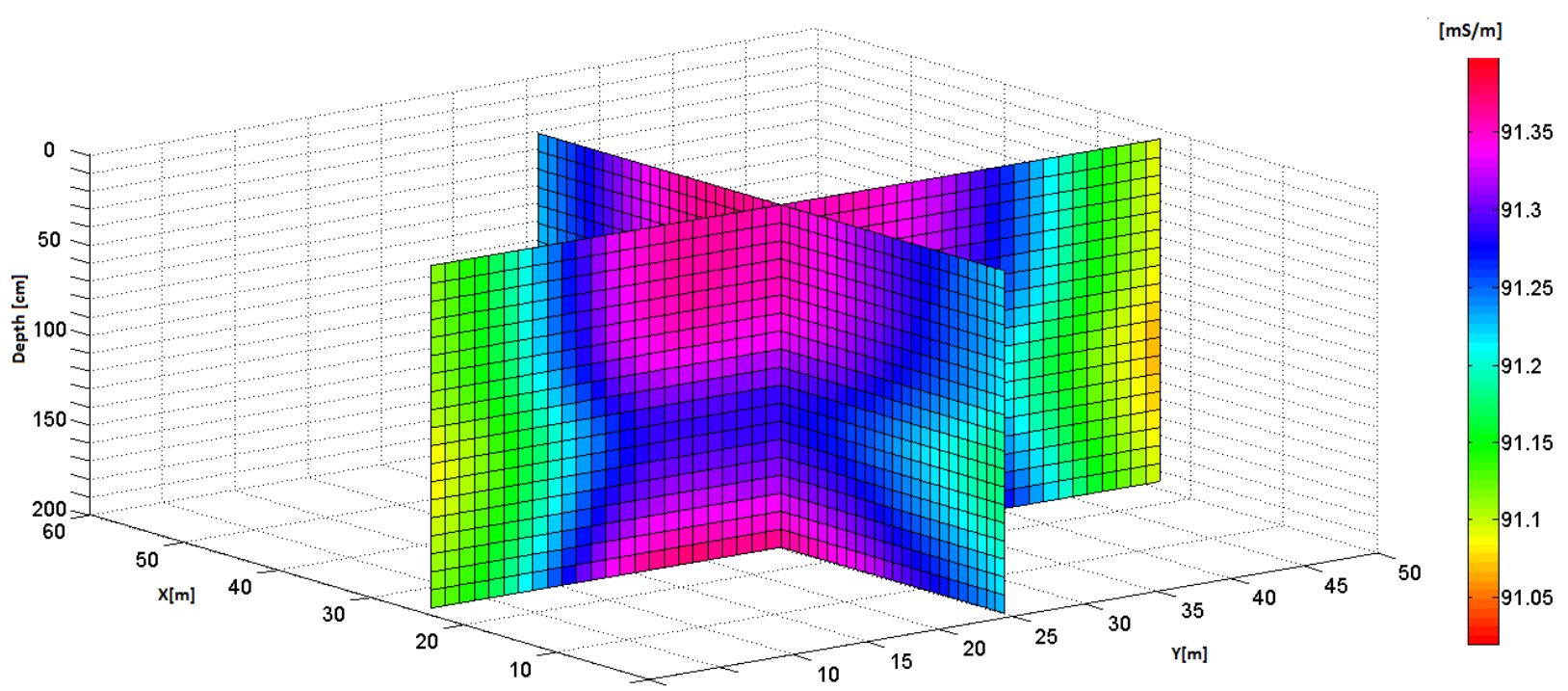

Figure 10. Electrical conductivity section in clay soil textures. 


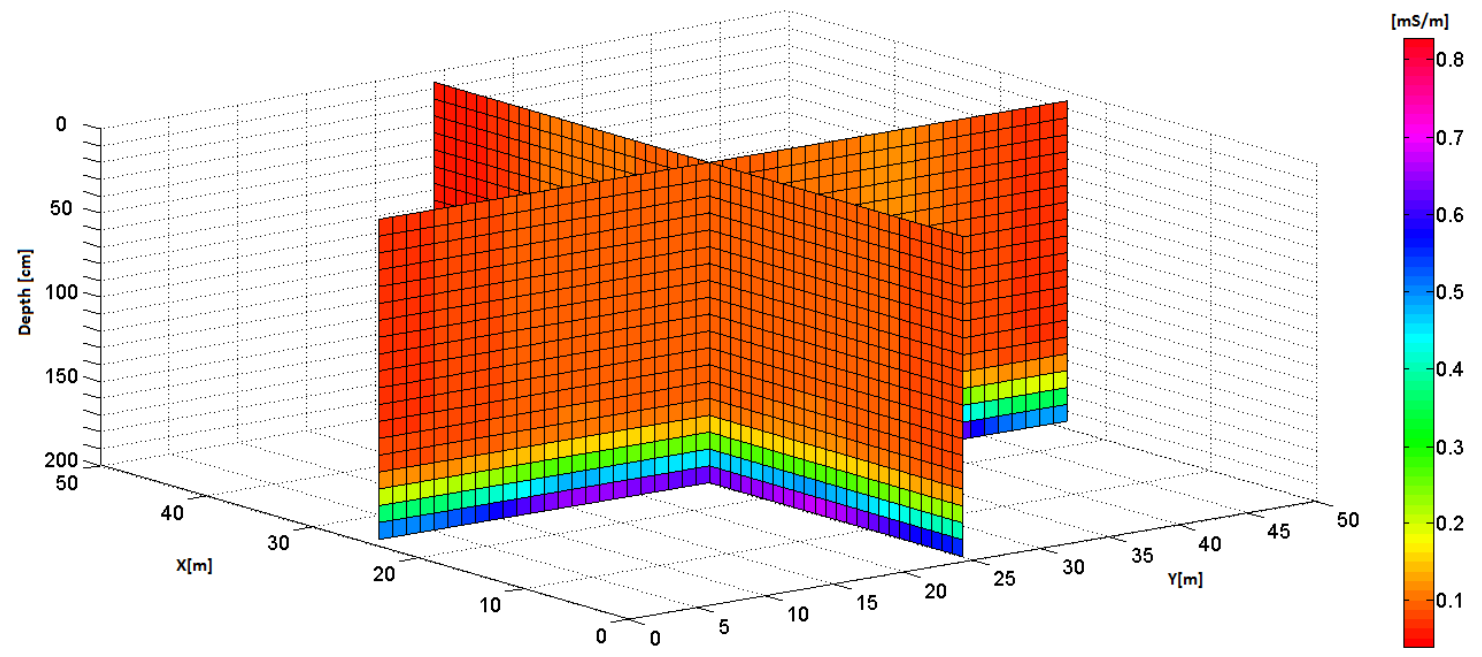

Figure 11. Electrical conductivity section in sand soil textures.

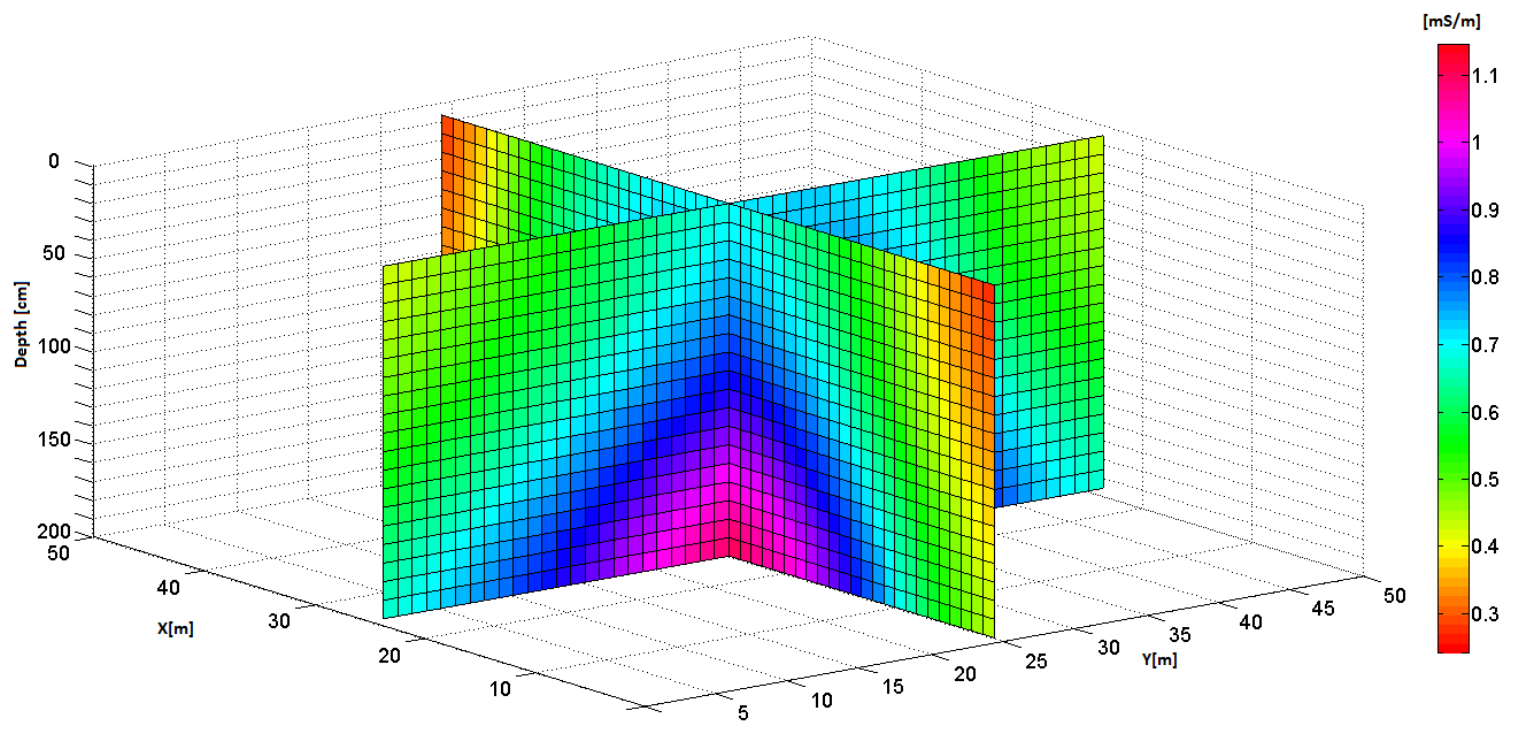

Figure 12. Electrical conductivity section in silt soil textures.

in a typical rain water electrical conductivity value of about $5 \mathrm{mS} / \mathrm{m}$. Furthermore, in the specific case of clay model, the contribution of the surface grains or particle conductivity (of about $90.94 \mathrm{mS} / \mathrm{m}$ ) has been taken into consideration. Clay surface's mineral particles influence considerably the electrical conductivity of this specific texture which results in an increase of the total current flow through soil as shown in Figure 10 and Table 4.

\section{Conclusions}

This paper dealt with the estimation of relative permittivity and electrical conductivity profiles inferred from SWC models for different soil textures. As well known the different percentages of the soil components, namely sand, clay and silt, described trough a numerical model allows to solve the Richards diffusion equa- tion and determine the SWC in dependence of depth and time. Such quantity is mainly responsible for the values assumed by the relative permittivity and conductivity along depth and time. Because of seldom geophysicists have a prior knowledge of the subsoil electrical parameters even in the case of homogeneous soil's composition these simulation yields of SWC along the vadose zone once recognized some characteristic properties of soil textures. Conversely, more commonly, this information can be used in the opposite sense inferring the SWC from the relative permittivity and conductivity. The prior knowledge of the permittivity and conductivity modelled profiles could help geophysicists and operators to better interpret the field measurement's results as in application of landfill monitoring where the soil textures are known. The modelled SWC and corresponding electric parameters are useful to choice the suitable tech- 


\begin{tabular}{cccc}
\hline $\begin{array}{c}\boldsymbol{\theta} \\
{\left[\mathrm{cm}^{3} / \mathrm{cm}^{3}\right]}\end{array}$ & $\begin{array}{c}\sigma \text { Sand } \\
{[\mathrm{mS} / \mathrm{m}]}\end{array}$ & $\begin{array}{c}\sigma \text { Silt } \\
{[\mathrm{mS} / \mathbf{m}]}\end{array}$ & $\begin{array}{c}\sigma \text { Clay } \\
{[\mathrm{mS} / \mathrm{m}]}\end{array}$ \\
\hline 0.1 & $9.010 \mathrm{E}-05$ & $7.680 \mathrm{E}-05$ & $9.094 \mathrm{E}+01$ \\
0.15 & $2.026 \mathrm{E}-04$ & $1.728 \mathrm{E}-04$ & $9.094 \mathrm{E}+01$ \\
0.2 & $3.603 \mathrm{E}-04$ & $3.072 \mathrm{E}-04$ & $9.094 \mathrm{E}+01$ \\
0.25 & $5.629 \mathrm{E}-04$ & $4.800 \mathrm{E}-04$ & $9.094 \mathrm{E}+01$ \\
0.3 & $8.106 \mathrm{E}-04$ & $6.912 \mathrm{E}-04$ & $9.094 \mathrm{E}+01$ \\
0.35 & $1.103 \mathrm{E}-03$ & $9.409 \mathrm{E}-04$ & $9.094 \mathrm{E}+01$ \\
0.4 & $1.441 \mathrm{E}-03$ & $1.229 \mathrm{E}-03$ & $9.094 \mathrm{E}+01$ \\
0.45 & $1.824 \mathrm{E}-03$ & $1.555 \mathrm{E}-03$ & $9.094 \mathrm{E}+01$ \\
0.5 & $2.252 \mathrm{E}-03$ & $1.920 \mathrm{E}-03$ & $9.094 \mathrm{E}+01$ \\
\hline
\end{tabular}

Table 4. Electrical conductivity and corresponding SWC values according to Archie relation.

niques to be employed and the optimal mathematical inversion algorithm to retrieve the electric profiles. The results of these simulations as reported in Tables 3 and 4, and Figures 7, 8 and 9 show the strong dependence of the permittivity from the SWC.

Other soil parameters have negligible influence on this quantity. Different is the case in which we consider the conductivity. In fact SWC dependence is important even if salinity, temperature, etc. are less relevant but still important for soil conductivity. The estimation of this two profiles have relevance especially when the soil textures are well characterised. In such a case this allows to simulate SWC through the above discussed model and consequently estimate these two electric quantities in function of depth and time in unsaturated soil. A comparison with others studies carried out previously [Zhou et al. 2001, Michot et al. 2003, Schwartz et al. 2008], showed the validity of the inverse method to convert soil moisture content to electrical resistivity using ERT with a modified form of the Archie's law. Starting from a given water content, electrical resistivity trend in time can be traced. Application in landfill monitoring is one the fruitful application since leachate dispersion can be profitably inferred through the measurements of the soil electric parameters $\varepsilon_{r}$ and $\sigma$.

\section{References}

Abidin, M.H.Z., F. Ahmad., D.C. Wijeyesekera., R. Saad and M.F.T. Baharuddin (2013). Soil Resistivity Measurements to Predict Moisture Content and Density in Loose and Dense Soil, Applied Mechanics and Materials, 353/356, 911-917.

Bianchi, F., M. Chiappini and R. Giordano (2015). Formazione, diffusione e distribuzione del percolato: modelli matematici integrati del progetto SIGLOD (Formation and distribution of leachate: integrated mathematical models of SIGLOD project), Quader- ni di Geofisica, 128, 25 p., ISSN 1590-2595, (in Italian). Bouksila, F., M. Persson, M.R. Berndtsson and A. Bahri (2008). Soil water content and salinity determination using different dielectric methods in saline gypsiferous soil, Hydrological Sciences Journal, 53 (1), 253-265.

Brooks, R.H., and A.T. Corey (1964). Hydraulic properties of porous media, Hydrology Papers, 3, Colorado State University, Fort Collins, Colorado.

Cosenza, P., C. Camerlynck and A. Tabbagh (2003). Differential effective medium schemes for investigating the relationship between high-frequency relative dielectric permittivity and water content of soils, Water Resources Research, 39 (9).

Cosenza, P., and A. Tabbagh (2004). Electromagnetic determination of clay water content: role of the microporosity, Applied Clay Science, 26, 21-36.

Dalton, F.N. (1992). Development of time-domain reflectrometry for measuring soil water content and bulk soil electrical conductivity, In: G.C. Topp and W.D. Reynolds (eds.), Advances in Measurement of Soil Physical Properties: Bringing Theory into Practice, Soil Science Society of America Spec. Publ. no. 30, Madison, Wisconsin, USA, 143-167.

Dannowski, G., and U. Yaramanci (1999). Estimation of water content and porosity using combined radar and geoelectrical measurements, Technical University of Berlin, Dept. of Applied Geophysics, Ackerstr. 71-76, D-13355 Berlin, Germany.

Griffiths, D.H., and R.F. King (1981). Applied Geophysics for Geologist and Engineers, The Element of Geophysical Prospecting, Pergamon Press, Oxford.

Hamed, Y., S. Ghada and M. Persson (2006). Evaluation of the WET sensor compared to time domain Reflectometry, Hydrological Sciences Journal, 51 (4), 671-681.

Huisman J.A., S.S. Hubbard., J.D. Redman and A.P. 
Annan (2003). A Measuring Soil Water Content with Ground Penetrating Radar: A Review, Vadose Zone Journal, 2, 476-491.

Kriaa, A., M. Hajjib, F. Jamoussib and A.H. Hamzaouib (2014). Electrical Conductivity of $1: 1$ and 2:1 Clay Minerals, Surface Engineering and Applied Electrochemistry, 50 (1), 84-94.

Ledieu, J., P. De Ridder, P. De Clerk and S. Dautrebande (1986). A Method of measuring soil moisture by Time Domain Reflectometry, Journal of Hydrology, 88, 319-328.

Leij, F.J., W.J. Alves., M.Th. van Genuchten and J.R. Williams (1996). The UNSODA unsaturated hydraulic database. EPA/600/R-96/095, U.S. Environmental Protection Agency, Cincinnati, $\mathrm{OH}$.

Leij, F.J., W.B. Russell and S.M. Lesch (1997). ClosedForm expression For Water Retention and conductivity data, Ground Water, 35 (5), 848-858.

Lesmes, D.P., and P. Shmulik Friedman (2005). Relationships between the electrical and hydrogeological properties of rocks and soils, In: Y. Rubin and S.S. Hubbard (eds.), Hydrogeophysics, Springer, Dordrecht, The Netherlands, Ch. 4, 87-128.

Malicki, M.A., R. Plagge and C.H Roth (1996). Improving the calibration of dielectric TDR soil moisture determination taking into account the solid soil, European Journal of Soil Science, 47, 357-366.

Michot, D., Y. Benderitter., A. Dorigny, B. Nicoullaud, D. King and A. Tabbagh (2003). Spatial and temporal monitoring of soil water content with an irrigated corn crop cover using electrical resistivity tomography, Water Resources Research, 39, 1138.

Mualem, Y. (1976). A new model for predicting the hydraulic conductivity of unsaturated porous media, Water Resources Research, 12 (3), 513-522.

Mukhlisin, M., and A. Saputra (2013). Performance Evaluation of Volumetric Water Content and Relative Permittivity Models, The ScientificWorld Journal, Vol. 2013, Article ID 421762, 7 p.

Nadler, A., A. Gamliel and I. Peretz (1999). Practical aspects of salinity effect on TDR-measured water content: a field study, Soil Science Society of America Journal, 63, 1070-1076.

Persson, M., R. Berndtsson, S. Nasri, J. Albergel, P. Zante and Y. Yumegaki (2000). Solute transport and water content measurements in clay soils using time domain reflectometry, Hydrological Sciences Journal, 45, 833-847.

Persson, M., B. Sivakumar, R. Berndtsson, O.H. Jacobsen and P. Schjønning (2002). Predicting the dielectric constant-water content relationship using artificial neural networks, Soil Science Society of America Journal, 66, 1424-1429.
Richards, L.A. (1931). Capillary conduction of liquids through porous mediums, Physics, 1 (5), 318-333.

Roth, K., R. Schulin, H. Fluhler and W. Attinger (1990). Calibration of time domain reflectometry for water content measurement using a composite dielectric approach, Water Resources Research, 26, 2267-2273.

Schwartz, B.F., M.E. Schreiber, P.S. Pooler and J.D. Rimstidt (2008). Calibrating access-tube time domain reflectometry soil water measurements in deep heterogeneous soils, Soil Science Society of America Journal, 72 (4), 917-930.

Settimi, A. (2011). Performance of electrical spectroscopy using a RESPER probe to measure salinity and water content of concrete and terrestrial soil, Annals of Geophysics, 54 (4), 400-413.

Shah, P.H., and D.N. Singh (2005). Generalized Archie’s Law for Estimation of Soil Electrical Conductivity, Journal of ASTM International, 2 (5), 1-20.

Telford, W.M., L.P. Geldart and R.E. Sheriff (1990). Applied Geophysics, 2nd ed., Cambridge University Press, Cambridge, 792 p.

Topp, G.C., J.L. Davis and A.P. Annan (1980). Electromagnetic determination of soil water content: Measurements in coaxial transmission lines, Water Resources Research, 16, 574-582.

Tuller, M., and D. Or (2005). Retention of water in soil and the soil water characteristic curve, In: D. Hillel (ed.), Encyclopedia if Soils in the Environment, Elsevier.

van Dam, R.L., B. Borchers and J.M.H. Hendrickx (2005). Methods for prediction of soil dielectric properties: a review, In: Proceedings of SPIE - The International Society for Optical Engineering 5794, 188-197.

van Genuchten, M.Th. (1980). A closed-form equation for predicting the hydraulic, conductivity of unsaturated soils, Soil Science Society of America Journal, 44, 892-898.

Zhou, Q.Y., J. Shimada and A. Sato (2001). Three-dimensional spatial and temporal monitoring of soil water content using electrical resistivity tomography, Water Resources Research, 37 (2), 273-285.

${ }^{\star}$ Corresponding author: Roberta Porretta, Istituto Nazionale di Geofisica e Vulcanologia, Rome, Italy; email: roberta.pr@live.com.

(C) 2016 by the Istituto Nazionale di Geofisica e Vulcanologia. All rights reserved. 